\title{
Publisher's Note: Probing the properties of event-by-event distributions in Hanbury-Brown-Twiss radii [Phys. Rev. C 92, 044906 (2015)]
}

Christopher Plumberg and Ulrich Heinz

(Received 16 October 2015; published 27 October 2015)

DOI: 10.1103/PhysRevC.92.049901

PACS number(s): 12.38.Mh, 24.10.Nz, 25.75.Gz, 99.10.Fg

This paper was published online on 13 October 2015 with an incorrect figure. Figure 2 has been replaced as of 16 October 2015. The figure is correct in the printed version of the journal. 